\title{
COVID-19 and threats to pregnancy and the fetus
}

\section{Przemysław Raczkiewicz, Natalia Śmiech, Tomasz Korzec, Konrad Kania, Marlena Kaczerska}

Medical University of Lublin, Poland

\begin{abstract}
11 March 2020 WHO assessmented that COVID-19 can be characterized as a pandemic. SARS-CoV-2 RNA virus is highly contagious and is transmitted by droplets.

It appears that pregnant women may be at a higher risk of being infected with SARS-CoV-2, which increases the risk to pregnancy and fetal defects. The course of the disease in this group of patients is more severe due to the changes in the immune and respiratory systems. Despite the constantly growing number of publications on the course of COVID-19 infection, the available data is insufficient.
\end{abstract}

Key words: COVID-19; SARS-CoV-2; pregnancy; childbirth; fetus. 


\section{Introduction}

COVID-19 is a disease caused by the SARS-CoV-2 RNA virus. In December 2019, a group of patients with pneumonia of unknown etiology with features of respiratory failure, fever and dry cough was reported in Wuhan. This virus is highly infectious and is transmitted by droplets from person to person. Most of those infected go through the disease in a mild manner, only in some cases in elderly patients and in those with chronic comorbidities the infection leads to serious consequences.

It appears that pregnant women may be at a higher risk of being infected with SARS-CoV-2, which increases the risk to pregnancy and fetal defects. The course of the disease in this group of patients is more severe due to the physiological changes in the immune and respiratory systems during pregnancy.

Despite the constantly growing number of publications on the course of COVID-19 infection in pregnant women, the available data is still limited and many questions remain unanswered.

\section{Purpose}

The constantly growing number of new infections with the SARS-CoV-2 virus in Poland and the related widespread anxiety among pregnant women prompts us to try to organize information on the risk of COVID-19. The purpose of this review was to present the literature data and the latest recommendations on risks to pregnancy, the fetus and maternal care.

\section{Method}

The latest literature was reviewed based on PubMed and Google Scholar databases, using the following keywords: COVID-19; SARS-CoV-2; pregnancy; childbirth; fetus.

\section{Results \\ Birth defects}

Pregnant women are more susceptible to viral infections, and therefore constitute a potential risk group of serious sequelae related to viral infections [1]. Physiological changes in the respiratory system of pregnant women occur, which facilitate the inhalation of infectious particles and may increase the risk of SARS-CoV-2 infection. There is a decrease in the level of Th1 cells (secreting pro-inflammatory cytokines: INF-gamma, IL-1a, IL-1b, IL6 , IL-12) and an increase in the level of Th2 cells (secreting anti-inflammatory cytokines: IL4, IL-10, IL-13, TGF-beta). These changes, on the one hand, protect the fetus with genetic material alien to the mother against rejection, but on the other hand, they increase the risk of infection [6]. In pregnant women, previous coronavirus outbreaks such as Middle East Respiratory Syndrome (MERS) and Severe Acute Respiratory Syndrome (SARS) have been associated with increased morbidity, mortality, and adverse maternal obstetric outcomes [2]. So far, only a few documented cases of SARS have been reported in pregnant women. A Hong Kong case study of seven cases during the first trimester of pregnancy showed a pregnancy loss rate of 57\% in SARS-infected women [3].

The fetal organs develop in the first trimester of pregnancy, and maternal infections at this stage may cause a number of complications in the child [1]. In a cohort study of 1,019 women, there was no significant difference in the translucency of the nuchal scan in pregnant women in the first trimester with prior SARS-CoV-2 infection (18 patients) compared to women without SARS-CoV-2 infection. Moreover, no significantly increased risk of pregnancy loss was found in women with positive antibodies in the first trimester of pregnancy [5]. The study shows that the risk of birth defects in the first trimester is likely to be low. 


\section{Risk of transmission to the fetus}

Most coronavirus infections in newborns are asymptomatic, but some may develop lethargy, fever and respiratory symptoms [14] [15].An infected mother can transmit the COVID-19 virus by airborne droplets at birth, but the probability is very low [14] [20]. Earlier studies did not reveal vertical transmission of SARS-CoV-2 from mother to fetus, which was confirmed by negative test results in amniotic fluid, umbilical cord blood, vaginal swabs and breast milk [7] [9] [10] [11] [12]. In a systematic review by Dell Gatta et al. [8], which describes 51 pregnant women who tested positive for COVID-19, vertical transmission was completely rejected. In a research Fan C, Lei D, Fang C. [10] 2 patients were presented with a positive test for the coronavirus. The risk of vertical transmission of SARS-CoV-2 was assessed as low because RT-PCR tests did not detect SARS-CoV-2 in any of the samples.

Other studies say that vertical transmission after maternal primary infection may occur in the intrauterine environment via the placenta, during labor, or through ingestion or aspiration of cervico-vaginal secretions and postpartum during breastfeeding [10]. Spread of infection through the placenta is less likely than during vaginal delivery. During vaginal delivery can come exposure to maternal faeces $(30 \%$ of infected patients have positive fecal RT-PCR results) and to maternal airway secretions [14].

More recent studies [13] report transmission of COVID-19 from mother to newborn across the placenta less than 24 hours after birth. RT-PCR results for amniotic fluid and newborn (less than 24 hours after birth) were positive for COVID-19. This fact suggests the possibility of vertical transmission of this disease. On day 11 and 14 the COVID-19 test was negative. In the case of [16], the infection of the newborn was also described, which occurred in the womb. The main finding in this case is the positive result of the newborn's nasopharyngeal swab RTPCR already at 16 and 48 hours after delivery. Wang et al. [18] reported a newborn with a positive RT-PCR result after 36 hours of delivery in a 34-year-old woman diagnosed with COVID-19.

In the study of Pettirosso et al. [15], the possibility of vertical virus transmission was assessed by RT-PCR of neonatal discharge from the nasopharynx, placenta, umbilical cord blood, amniotic fluid and mother's milk. 19 out of a total of 655 nasopharyngeal smears of newborns were positive for SARS-CoV-2. In addition, 4 placenta samples and one umbilical cord blood sample were positive, however neonatal nose and throat swabs were negative in these cases. This suggests the possibility of vertical transmission, but it is not clear whether the infection occurs in utero, intrapartum or postnatal life.

\section{Passive immunization of the newborn by the mother}

Breastfeeding in a pandemic is a challenge as there is insufficient evidence to report on the risks. Recent studies suggest that newborns may benefit from breastfeeding [19] [20] [21]. No maternal milk samples were positive for SARS-CoV-2 and to date there is no conclusive evidence of the presence of the virus in milk of pregnant COVID-19 mothers [19]. Moreover, breastfeeding with appropriate protective measures (using a mask, washing hands before contact with a child) has a low risk of transmitting SARS-CoV-2 to the child [15].

In a 33-year-old woman in the 38th week of pregnancy suffering from cough and tightness in the chest, a throat swab was positive for SARS-CoV-2. The baby was born in an isolated delivery room and quarantined in the neonatal intensive care unit. An oropharyngeal swab taken immediately after birth was negative for the detection of SARS-CoV-2 RNA. The titres 
of IgG antibodies in breast milk were 2.34, 3.02, 2.84, 2.79 and 3.35 (control test 0.98 ). The titres of IgA antibodies in breast milk were 2.18, 2.23, 2.37 and 2.69 (control test 0.9) [21].

Milk samples were tested for antibodies in 4 mothers infected with the coronavirus. Three samples tested positive for IgM or IgG for SARS-CoV-2 [20].

The mother can passively immunize the baby by transmitting IgG antibodies across the placenta from the end of the second trimester. They reach a high level at birth. IgM is usually not transferred from mother to fetus due to its size [22]. Among 6 mothers with confirmed COVID-19, SARS-CoV-19 was not detected in serum or throat swab via RT-PCR in any of their newborns. All of them had Caesarean sections in the third trimester of pregnancy in vacuum isolators. All mothers wore masks, and all medical personnel wore protective suits and double masks. Infants were isolated from their mothers immediately after delivery. Serum IgG concentrations were increased in 5 infants, which confirms passive immunization of newborns by mothers [22]. Increased IgM was detected in 2 infants, which may suggest their production by the fetus following vertical infection or damage to the placenta [23].

\section{Recommendations for pregnant women}

Since the announcement of the COVID-19 pandemic by the WHO, many scientific societies have issued their own recommendations for the management of pregnant women [24] [25]. It is recommended that:

- All pregnant women should take extensive preventive measures: hand hygiene and surface disinfection with a product containing more than $60 \%$ ethanol, and strictly observe social distancing when interacting with other people. This also applies to their partners.

- Personal visits should be minimized in favor of medical visits and home visits if the patient shows no symptoms of illness.

- For pregnant women working in places with a high risk of infection (operating theaters, pulmonary departments, intensive care), it is preferable to transfer to places with lower exposure. Pregnant patients over 24 weeks of age should be strictly protected against infection, because treatment of severe pneumonia in pregnancy, intubation and mechanical ventilation are quite difficult, especially in the third trimester of pregnancy.

- The number of visits to hospital by women should be limited, and some guidelines suggest limiting it to one visit per day. Women with suspected or confirmed COVID-19 should not receive visitors.

- Fetal growth should be monitored in pregnant women infected with COVID-19. Additional ultrasound evaluation at the gestational age of 24-28-32-36 weeks is recommended, including the evaluation of biometry, measurement of amniotic fluid volume and evaluation of blood flow in the uterine arteries.

- It is unlikely that your baby will become infected while passing through the birth canal, but the available data are limited.

- The benefits of breastfeeding (with preventive measures such as wearing a surgical mask, hand hygiene, and washing the nipples before breastfeeding) outweigh the possible risk of infection in the newborn. Consider expressing milk and bottle feeding. 
- Skin-to-skin contact between mother and newborn should be maintained regardless of maternal SARS-CoV-2 infection, provided that appropriate personal protective equipment is worn.

- Pregnant patients should be evaluated for the severity of respiratory symptoms and the possible presence of comorbidities. In severe, critical cases, immediate referral to a tertiary reference center is indicated.

- Corticosteroids are important in the prevention of neonatal lung hypoplasia, necrotizing enteritis, and prematurity-induced interventricular haemorrhage. Some publications suggest a careful risk-benefit assessment before starting therapy.

- Antiretroviral drugs are currently being tested in clinical trials. They cannot be used during pregnancy, except when there are no other treatment options to protect the mother's life, and should be selected on an individual basis.

- In pregnant women with COVID-19, antibiotic therapy should only be used when there is evidence of secondary bacterial infection.

\section{Conclusions}

Unlike SARS and MERS, pregnancy does not appear to be associated with an increased risk of serious COVID-19 disease in the mother compared to the non-pregnant population.Vertical transmission of COVID-19 is under discussion and clinical evidence is extremely sparse and based on a small number of reports and case reports. Research results suggest that passive acquisition of anti-SARS-CoV-2 antibodies is possible through the consumption of breast milk and as a result of the transmission of IgG antibodies across the placenta. Among the published recommendations, there was a consensus on specific actions and interventions. The greatest differences are in the guidelines for corticosteroid use, neonatal isolation after birth, and disease-modifying drugs. Divergences between the different organizations developing the guidelines pose a challenge for healthcare professionals involved in maternity care during the COVID-19 pandemic.

\section{Bibliography}

[1] Silasi, M., Cardenas, I., Kwon, J.-Y., Racicot, K., Aldo, P., \& Mor, G. (2015). Viral Infections During Pregnancy. American Journal of Reproductive Immunology, 73(3), 199213. doi: https://doi.org/10.1111/aji.12355

[2] Schwartz, \& Graham. (2020). Potential Maternal and Infant Outcomes from (Wuhan) Coronavirus 2019-nCoV Infecting Pregnant Women: Lessons from SARS, MERS, and Other Human Coronavirus Infections. Viruses, 12(2), 194. doi: https://doi.org/10.3390/v12020194

[3] Wong, S. F., Chow, K. M., Leung, T. N., Ng, W. F., Ng, T. K., Shek, C. C., ... Tan, P. Y. . (2004). Pregnancy and perinatal outcomes of women with severe acute respiratory syndrome. American Journal of Obstetrics and Gynecology, 191(1), 292-297. doi: https://doi.org/10.1016/j.ajog.2003.11.019

[4] Assiri, A., Abedi, G. R., Al Masri, M., Bin Saeed, A., Gerber, S. I., \& Watson, J. T. (2016). Middle East Respiratory Syndrome Coronavirus Infection During Pregnancy: A Report of 5 Cases From Saudi Arabia: Table 1. Clinical Infectious Diseases, 63(7), 951-953. doi: https://doi.org/10.1093/cid/ciw412

[5] La Cour Freiesleben, N., Egerup, P., Vauvert Römmelmayer Hviid, K., Rosenbek Severinsen, E., Kolte, A. M., Westergaard, D., ... Nielsen, H. S. (2020). SARS-CoV-2 in first 
trimester pregnancy: a cohort study. Human Reproduction. doi: https://doi.org/10.1093/humrep/deaa311

[6] Zhao, X., Jiang, Y., Zhao, Y., Xi, H., Liu, C., Qu, F., \& Feng, X. (2020). Analysis of the susceptibility to COVID-19 in pregnancy and recommendations on potential drug screening. European Journal of Clinical Microbiology \& Infectious Diseases, 39(7), 1209-1220. doi: https://doi.org/10.1007/s10096-020-03897-6

[7] Dashraath, P., Wong, J. L. J., Lim, M. X. K., Lim, L. M., Li, S., Biswas, A., ... \& Su, L. L. (2020). Coronavirus disease 2019 (COVID-19) pandemic and pregnancy. American journal of obstetrics and gynecology, 222(6), 521-531. doi: https://doi.org/10.1016/j.ajog.2020.03.021

[8] Della Gatta, A. N., Rizzo, R., Pilu, G., \& Simonazzi, G. (2020). COVID19 during pregnancy: a systematic review of reported cases. American Journal of Obstetrics and Gynecology, 223(1): 36-41. doi: https://doi.org/10.1016/j.ajog.2020.04.013

[9] Chen, H., Guo, J., Wang, C., Luo, F., Yu, X., Zhang, W., ... Zhang, Y. (2020). Clinical characteristics and intrauterine vertical transmission potential of COVID-19 infection in nine pregnant women: a retrospective review of medical records. The Lancet, 395(10226), 809-815. doi: https://doi.org/10.1016/s0140-6736(20)30360-3

[10] Cuifang Fan, Di Lei, Congcong Fang, Chunyan Li, Ming Wang, Yuling Liu, Yan Bao, Yanmei Sun, Jinfa Huang, Yuping Guo, Ying Yu, Suqing Wan. (2021). Perinatal Transmission of 2019 Coronavirus Disease-Associated Severe Acute Respiratory Syndrome Coronavirus 2: Should We Worry? Clinical Infectious Diseases, 72(5), 862-864. doi: https://doi.org/10.1093/cid/ciaa226

[11] Yang, H., Wang, C., \& Poon, L. C. (2020). Novel coronavirus infection and pregnancy. Ultrasound in Obstetrics \& Gynecology, 55(4), 435-437. doi: https://doi.org/10.1002/uog.22006

[12] Li, Y., Zhao, R., Zheng, S., Chen, X., Wang, J., Sheng, X., ... Sheng, J. (2020). Lack of Vertical Transmission of Severe Acute Respiratory Syndrome Coronavirus 2, China. Emerging Infectious Diseases, 26(6). 1335. doi: https://doi.org/10.3201/eid2606.200287

[13] Yekta Parsa, Nazila Shokri, Tayebeh Jahedbozorgan, Zahra Naeiji, Shahrzad Zadehmodares, Atefeh Moridi. (2021). Possible Vertical Transmission of COVID-19 to the Newborn; a Case Report. Archives of Academic Emergency Medicine, 9(1). https://doi.org/10.22037/aaem.v9i1.923

[14] Lamouroux, A., Attie-Bitach, T., Martinovic, J., Leruez-Ville, M., \& Ville, Y. (2020). Evidence for and against vertical transmission for severe acute respiratory syndrome coronavirus 2. American Journal of Obstetrics and Gynecology, 223(1), 91-e1. doi: https://doi.org/10.1016/j.ajog.2020.04.039

[15] Pettirosso, E., Giles, M., Cole, S., \& Rees, M. (2020). COVID-19 and pregnancy: A review of clinical characteristics, obstetric outcomes and vertical transmission. Australian and New Zealand Journal of Obstetrics and Gynaecology. 60(5), 640-659. doi: https://doi.org/10.1111/ajo.13204

[16] Alzamora, M. C., Paredes, T., Caceres, D., Webb, C. M., Valdez, L. M., \& La Rosa, M. (2020). Severe COVID-19 during Pregnancy and Possible Vertical Transmission. American Journal of Perinatology, 37(08): 861-865. doi: https://doi.org/10.1055/s-0040-1710050 
[17] Prochaska, E., Jang, M., \& Burd, I. (2020). COVID-19 in pregnancy: Placental and neonatal involvement. American Journal of Reproductive Immunology, 84:e13306. doi: https://doi.org/10.1111/aji.13306

[18] Wang, S., Guo, L., Chen, L., Liu, W., Cao, Y., Zhang, J., \& Feng, L. (2020). A case report of neonatal COVID-19 infection in China. Clinical Infectious Diseases, 71(15):853-857. doi: https://doi.org/10.1093/cid/ciaa225

[19] Martins-Filho, P. R., Santos, V. S., \& Santos, H. P. (2020). To breastfeed or not to breastfeed? Lack of evidence on the presence of SARS-CoV-2 in breastmilk of pregnant women with COVID-19. Revista Panamericana de Salud Pública, 44, 1-7. doi: https://doi.org/10.26633/rpsp.2020.59

[20] Gao, X., Wang, S., Zeng, W., Chen, S., Wu, J., Lin, X., ... Feng, L. (2020). Clinical and immunological features among COVID-19 affected mother-infant pairs: antibodies to SARSCoV- 2 detected in breast milk. New Microbes and New Infections, 37, 100752. doi: https://doi.org/10.1016/j.nmni.2020.100752

[21] Dong, Y., Chi, X., Huang, H., Sun, L., Zhang, M., Xie, W.-F., \& Chen, W. (2020). Antibodies in the breast milk of a maternal woman with COVID-19. Emerging Microbes \& Infections, 9(1), 1467-1469. doi: https://doi.org/10.1080/22221751.2020.1780952

[22] Zeng, H., Xu, C., Fan, J., Tang, Y., Deng, Q., Zhang, W., \& Long, X. (2020). Antibodies in Infants Born to Mothers With COVID-19 Pneumonia. JAMA, 323(18), 1848-1849. doi: https://doi.org/10.1001/jama.2020.4861

[23] Chen, Huang ,Luo D,Li ,Yang,Zhao,Nie , Huang. (2020). [Pregnancy with new coronavirus infection: clinical characteristics and placental pathological analysis of three cases]. Chinese Journal of Pathology, 49(5), 418-423. Chinese. PMID: 32114744. doi: https://doi.org/10.3760/cma.j.cn112151-20200225-00138

[24] Pavlidis, P., Eddy, K., Phung, L., Farrington, E., Connolly, M., Lopes, R., ... Vogel, J. P. (2020). Clinical guidelines for caring for women with COVID-19 during pregnancy, childbirth and the immediate postpartum period. Women and Birth. In press. doi: https://doi.org/10.1016/j.wombi.2020.10.015

[25] Donders, F., Lonnée-Hoffmann, R., Tsiakalos, A., Mendling, W., Martinez de Oliveira, J., Judlin, P., Xue, F., Donders, G., \& Isidog Covid-Guideline Workgroup (2020). ISIDOG Recommendations Concerning COVID-19 and Pregnancy. Diagnostics (Basel, Switzerland), 10(4), 243. https://doi.org/10.3390/diagnostics 10040243 